\title{
Corneal Irregular Astigmatism And Visual Function On Anterior Segment Optical Coherence Tomography In TGFBI Corneal Dystrophy
}

\author{
Yuito Abe \\ University of Tokyo Graduate School of Medicine \\ Takashi Omoto ( $\square$ oomoto-tky@umin.ac.jp ) \\ University of Tokyo Graduate School of Medicine \\ Kohdai Kitamoto \\ University of Tokyo Graduate School of Medicine \\ Tetsuya Toyono \\ University of Tokyo Graduate School of Medicine \\ Junko Yoshida \\ International University of Health and Welfare \\ Ryo Asaoka \\ Seirei Hamamatsu General Hospital \\ Satoru Yamagami \\ Nihon University School of Medicine \\ Takashi Miyai \\ University of Tokyo Graduate School of Medicine \\ Tomohiko Usui \\ International University of Health and Welfare
}

\section{Research Article}

Keywords:

Posted Date: January 21st, 2022

DOI: https://doi.org/10.21203/rs.3.rs-1251041/v1

License: (9) (1) This work is licensed under a Creative Commons Attribution 4.0 International License. Read Full License 


\section{Abstract}

The purpose of this study was to evaluate corneal irregular astigmatism of patients with granular and lattice corneal dystrophy (GCD and LCD). 70 GCD, 35 LCD, and 23 control eyes were included. Anterior and posterior corneal topographic data obtained from anterior segment optical coherence tomography were expanded into four components via Fourier harmonic analysis. These components were compared with healthy eyes and the association between each component and best-corrected visual acuity (BCVA) was investigated. Anterior and posterior components increased in LCD eyes, but none increased in GCD. Posterior components of GCD type 2 (GCD2), anterior and posterior of LCD type 1 (LCD1), posterior of LCD type IIIA (LCD 3A) and type IV (LCD4) significantly increased. BCVA was significantly associated with anterior and posterior components in LCD eyes but not in GCD. The anterior components of LCD1, anterior and posterior of LCD3A, and posterior of LCD4, but not the posterior of LCD3A, were positively correlated with BCVA. As conclusions, in GCD eyes, none of the components differed from those of the control and BCVA was not significantly associated with them. In LCD eyes, the anterior and posterior components increased, and BCVA was significantly associated with the anterior and posterior components.

\section{Introduction}

Transforming growth factor beta-induced (TGFBI) corneal dystrophy is a bilateral corneal disease characterized by an abnormal

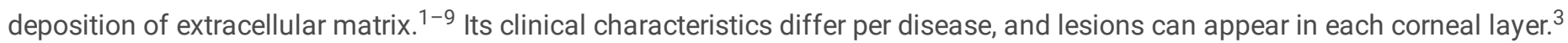
Patients with TGFBI dystrophy present with reduced best-corrected visual acuity (BCVA) with disease progression. However, the severity of opacity and visual function might not always be correlated. ${ }^{10,11}$

With recent technological advancements, images and accurate information about the cornea and anterior segment of the eyes can be obtained. Corneal irregularities are evaluated by analyzing wavefront aberrations, which can explain the decrease in BCVA and contrast sensitivity in normal and diseased eyes. ${ }^{10-18}$ Recently, with the advent of anterior segment optical coherence tomography (AS-OCT) using infrared light with high depth of field, irregular astigmatisms in the anterior and posterior surfaces, even in cloudy corneas, can now be quantified. Fourier analysis is performed to quantify the shape analysis of the anterior segment, which can explain low BCVA and contrast sensitivity. ${ }^{19}$

Stromal opacity and a higher-order aberration (HOA) of the cornea can affect BCVA in corneal dystrophy. ${ }^{10,20}$ The current study focused on granular corneal dystrophy (GCD) and lattice corneal dystrophy (LCD), which account for most TGFBI dystrophy cases. GCD and LCD are the same in that both are related to allelic mutations of the TGFBI gene, ${ }^{21-23}$ whereas they are known to show quite different findings in cornea; different types of deposits are observed in various layers. ${ }^{3}$ However, few have investigated the anterior and posterior surfaces of the cornea in TGFBI dystrophy and its different subtypes in detail. ${ }^{10}$ Therefore, in the current study, we aimed to evaluate anterior and posterior irregular astigmatism in GCD and LCD via a Fourier harmonic analysis of AS-OCT data.

\section{Results}

In total, 70 GCD eyes in 37, 35 LCD eyes in 21, and 32 control eyes in 23 participants were included in the current study. The GCD and LCD groups had significantly worse BCVA than the control group. Table 1 and Figure 1 show the characteristics of the participants and representative photos of the groups, respectively. 
Table 1

Demographic characteristics of the participants

\begin{tabular}{|c|c|c|c|c|c|c|c|c|}
\hline & \multirow[t]{2}{*}{ Controls } & \multicolumn{3}{|l|}{ GCD } & \multicolumn{4}{|l|}{ LCD } \\
\hline & & Total & GCD1 & GCD2 & Total & LCD1 & LCD3A & LCD4 \\
\hline $\begin{array}{l}\text { No. of eyes } \\
\text { (patients) }\end{array}$ & 32 (23) & 70 (37) & $4(2)$ & $66(35)$ & $35(21)$ & $11(7)$ & $11(6)$ & $13(8)$ \\
\hline Age (years) & $64.6 \pm 9.2$ & $\begin{array}{l}60.4 \pm \\
16.3\end{array}$ & $59.0 \pm 4.6^{*}$ & $\begin{array}{l}60.4 \pm \\
16.8\end{array}$ & $\begin{array}{l}66.9 \pm \\
14.9\end{array}$ & $\begin{array}{l}51.1 \pm \\
13.7 *\end{array}$ & $\begin{array}{l}75.8 \pm \\
7.8^{\star}\end{array}$ & $\begin{array}{l}72.6 \pm \\
9.9 *\end{array}$ \\
\hline $\begin{array}{l}\text { Sex } \\
\text { (male/female) }\end{array}$ & $14 / 18$ & $26 / 44$ & $0 / 4$ & $26 / 40$ & 24/11 & $6 / 5$ & $9 / 2^{*}$ & $9 / 4$ \\
\hline IOL eyes & 0 & 14 & 0 & 14 & 10 & 2 & 5 & 3 \\
\hline BCVA (LogMAR) & $\begin{array}{l}-0.064 \pm \\
0.031\end{array}$ & $\begin{array}{l}0.16 \pm \\
0.24^{*}\end{array}$ & $\begin{array}{l}-0.059 \pm \\
0.040\end{array}$ & $\begin{array}{l}0.17 \pm \\
0.23 *\end{array}$ & $\begin{array}{l}0.52 \pm \\
0.51 *\end{array}$ & $\begin{array}{l}0.69 \pm \\
0.58 *\end{array}$ & $\begin{array}{l}0.30 \pm \\
0.20 \star\end{array}$ & $\begin{array}{l}0.56 \pm \\
0.56 \star\end{array}$ \\
\hline \multicolumn{9}{|c|}{$\begin{array}{l}\text { GCD = granular corneal dystrophy, GCD1 = granular corneal dystrophy type } 1, \mathrm{GCD} 2 \text { = granular corneal dystrophy type } 2, \mathrm{LCD}= \\
\text { lattice corneal dystrophy, } \mathrm{LCD} 1=\text { lattice corneal dystrophy type } 1, \mathrm{LCD} 3 \mathrm{~A}=\text { lattice corneal dystrophy type } 3 \mathrm{~A}, \mathrm{LCD} 4=\mathrm{lattice} \\
\text { corneal dystrophy type } 4, \mathrm{IOL}=\text { intraocular lens, BCVA = best-corrected visual acuity. Values were shown in mean } \pm \text { standard } \\
\text { deviation manner. }\end{array}$} \\
\hline
\end{tabular}

In the analysis according to phenotypes, when comparing the GCD and control eyes, there was no significant difference in terms of the anterior and posterior components between the two groups (Figure 2). Furthermore, these components were not associated with BCVA (Table 2). In the comparison between the LCD and normal groups, the anterior (asymmetry) $(P=0.030)$ and all of the posterior components $(P<0.001)$ significantly differed (Figure 2$)$. The Fourier components of the LCD eyes were significantly associated with BCVA in the anterior (regular astigmatism, asymmetry, and higher-order irregularity; $\mathrm{P}=0.012,<0.001$, and $<0.001$ ) and posterior (higher-order irregularity; $\mathrm{P}=0.029$ ) components (Table 2).

Table 2. Association between BCVA and Fourier components

Coefficients and $p$ values were calculated using the linear mixed effect model. *, $p$ value $<0.05$ 


\begin{tabular}{|c|c|c|c|c|c|c|c|c|c|}
\hline & & Anterior & & & & Posterior & & & \\
\hline & & Spherical & Regular & Asymmetry & $\begin{array}{l}\text { Higher- } \\
\text { order } \\
\text { irregularity }\end{array}$ & Spherical & Regular & Asymmetry & $\begin{array}{l}\text { Higher- } \\
\text { order } \\
\text { irregularity }\end{array}$ \\
\hline \multirow[t]{2}{*}{ GCD } & Coefficient & -0.012 & 0.021 & 0.059 & -0.088 & 0.043 & -0.28 & 0.047 & -0.028 \\
\hline & $P$ value & 0.33 & 0.66 & 0.40 & 0.77 & 0.64 & 0.29 & 0.74 & 0.95 \\
\hline \multirow[t]{2}{*}{ GCD1 } & Coefficient & -0.056 & 0.029 & -0.17 & -1.32 & -0.30 & 0.23 & -0.70 & -3.96 \\
\hline & $P$ value & 0.82 & 0.90 & 0.50 & 0.50 & 0.82 & 0.78 & 0.66 & 0.50 \\
\hline \multirow[t]{2}{*}{ GCD2 } & Coefficient & -0.016 & 0.020 & 0.061 & -0.11 & 0.039 & -0.16 & 0.017 & -0.085 \\
\hline & P value & 0.20 & 0.67 & 0.40 & 0.72 & 0.66 & 0.58 & 0.90 & 0.84 \\
\hline \multirow[t]{2}{*}{ LCD } & Coefficient & -0.054 & 0.28 & 0.27 & 1.55 & 0.19 & 0.40 & -0.026 & 0.39 \\
\hline & $P$ value & 0.25 & $0.012^{*}$ & $<0.001^{\star}$ & $<0.001 *$ & 0.39 & 0.13 & 0.87 & $0.029 *$ \\
\hline \multirow[t]{2}{*}{ LCD1 } & Coefficient & -0.060 & 0.33 & 0.31 & 1.50 & 0.44 & -1.42 & -0.30 & -3.28 \\
\hline & $P$ value & 0.50 & $0.046^{*}$ & $<0.001^{\star}$ & 0.064 & 0.60 & 0.33 & 0.52 & 0.35 \\
\hline \multirow[t]{2}{*}{ LCD3 } & Coefficient & -0.042 & 0.35 & 0.47 & 3.30 & -0.030 & -2.32 & 0.46 & 0.70 \\
\hline & $P$ value & 0.69 & 0.30 & $0.047^{*}$ & $0.036^{*}$ & 0.95 & $0.0048 *$ & 0.24 & 0.12 \\
\hline \multirow[t]{2}{*}{ LCD4 } & Coefficient & -0.10 & 0.15 & 0.18 & 1.59 & 0.23 & 0.67 & 0.11 & 0.43 \\
\hline & $P$ value & 0.40 & 0.65 & 0.18 & 0.12 & 0.49 & $0.010^{*}$ & 0.64 & $0.023^{*}$ \\
\hline
\end{tabular}

$\mathrm{BCVA}=\mathrm{GCD}=$ granular corneal dystrophy, GCD1 = granular corneal dystrophy type 1, GCD2 = granular corneal dystrophy type 2, LCD $=$ lattice corneal dystrophy, LCD1 = lattice corneal dystrophy type 1, LCD3A = lattice corneal dystrophy type 3A, LCD4 = lattice corneal dystrophy type 4

In the analysis according to subtypes of the GCD group, the eyes of patients with GCD1 and controls were similar (Figure 3). There was no association between BCVA and each of the components (Table 2). However, the posterior components (asymmetry, higherorder irregularity) of GCD2 were significantly higher than those of the normal eyes $(P=0.0012,0.0071)$ (Figure 3 ). Nevertheless, the posterior components were not significantly correlated with BCVA (Table 2).

In the analysis according to LCD subtypes, the eyes with LCD1 had a higher anterior (regular astigmatism, asymmetry, and higherorder irregularity; $\mathrm{P}=<0.001,0.0064$, and 0.020 ) and posterior (spherical; $\mathrm{P}=0.045$ ) components (Figure 3 ). Moreover, the anterior (regular astigmatism, asymmetry; $\mathrm{P}=0.046,<0.001$ ) components were positively associated with BCVA (Table 2).

Only the posterior components of the eyes in patients with LCD3A significantly differed (spherical; $P=0.045$ ) (Figure 3 ). The anterior components (asymmetry, higher-order irregularity; $\mathrm{P}=0.047,0.036$ ) were positively associated with $\mathrm{BCVA}$. Meanwhile, there was a negative correlation between the posterior component (regular astigmatism; $\mathrm{P}=0.0048$ ) and BCVA (Table 2).

In LCD4, the anterior components did not significantly differ, and all posterior components increased $(P=0.0060,<0.001,<0.001$, and $<0.001$ ) (Figure 3 ). In addition, there was a positive association only between the posterior components (regular astigmatism, higherorder irregularity; $\mathrm{P}=0.010,0.023$ ) and BCVA (Table 2).

\section{Discussion}

The specific profile of the layer-by-layer Fourier components in GCD and LCD eyes, in addition to the association between them and BCVA, was discussed in this research. Moreover, not only each phenotype but also each subtype was analyzed. GCD and LCD are known to show quite different findings in cornea; both amyloid and hyaline deposits in GCD eyes, whereas amyloid deposits in LCD eyes and the depth of the deposits vary by the subtypes. ${ }^{3}$ These findings were analyzed using AS-OCT in detail and these results should be beneficial for clinicians when evaluating visual function of the patients. 
In GCD eyes, interstitial opacities between granular deposits were often confined to the anterior subepithelial layer (Figure 1). Structural disturbances in the anterior and posterior surfaces were apparently smooth. However, there was a slight but significant increase in posterior irregular astigmatism (asymmetry, higher-order irregularity) in GCD2 eyes (Figure 3). Interestingly, the posterior, but not the anterior, components increased despite the relatively superficial existence of opacity. In a previous report by Yagi-Yaguchi et al., visual acuity was positively correlated with opacity grade, age, astigmatism in GCD2 eyes, but not with HOAs. ${ }^{10}$ The HOA value in the correlation analysis in a previous study was not calculated layer by layer. ${ }^{10}$ The current study analyzed the anterior and posterior components separately. However, the results were similar. That is, the Fourier components of the GCD eyes were not correlated with BCVA, which did not change when analyzed according to each subtype. Nevertheless, the GCD eyes had significantly lower BCVA than the control eyes. The factors causing a decrease in BCVA are challenging to analyze. However, the relatively low contributions of astigmatism factors reinforce the possibility that scattering caused by opacity may have a stronger effect on visual acuities.

In LCD eyes, there was an increase in Fourier components, and BCVA in the anterior and posterior surfaces was positively associated with these components. In the analysis according to each LCD subtype, the results differed between the subtypes and were characteristic to each subtype. As shown in Figure 1, the cornea of one patient with LCD1 had stromal opacities with various reflectivity and sizes near the anterior surface. Previously, Yagi-Yaguchi et al. showed that visual acuity was correlated with HOAs, but not with grade, age, and astigmatism, in patients with LCD1, unlike GCD. ${ }^{10}$ The study results were similar. That is, there was an increase in anterior components (regular astigmatism, asymmetry, and higher-order irregularity), which were associated with BCVA.

As shown in Figure 1, the cornea of patients with LCD4 commonly presented with opacities in the posterior surface, which could be described as protrusions in the anterior chamber. In LCD4 eyes, all components of the posterior surface increased, and almost all were positively associated with BCVA. The results of the current research were consistent with those of previous studies. ${ }^{27,28}$

In LCD1, PTK is sometimes performed because of a significant anterior surface lesion. ${ }^{29}$ Nevertheless, in LCD4, corneal transplantation is still considered as the primary treatment as it can improve BCVA since the posterior irregularity cannot be corrected using rigid gas permeable contact lenses and the posterior opacity cannot be managed with PTK. Each subtype of LCD had different characteristics. In each subtype, the results of the corneal shape analysis were similar to the typical AS-OCT imaging findings and were consistent with what has been noted clinically.

The current study had several limitations. The degree of opacity was not evaluated. As mentioned above, the effect of scattering caused by opacity on visual function cannot be ignored. Thus, the quantified data about opacity and its association with BCVA must be investigated. Subtype classification was based on clinical, not genetic, diagnosis. Furthermore, some patients who underwent cataract surgery were included. The effect of cataract surgery on corneal shapes cannot been completely ruled out, ${ }^{30-32}$ even though these changes are temporary based on several reports. ${ }^{33-35}$

In conclusion, in GCD eyes, except for a slight increase in the posterior components of GCD2 based on a subtype analysis, none of the components differed from the control, and BCVA was not significantly associated with these components. In LCD eyes, the anterior and posterior components increased, and BCVA was significantly associated with the anterior and posterior components. In the analysis according to each LCD subtype, the results differed between the subtypes; both anterior and posterior components differed and anterior components positively associated with BCVA in LCD1, only the posterior components differed and the associations with BCVA were positive in anterior and negative in posterior components in LCD3A and posterior components increased and there was a positive association between posterior components and BCVA in LCD4.

\section{Methods}

This retrospective observational study was approved by the institutional review board of University of Tokyo Hospital (20200006NI). Informed consent was obtained in the form of opt-out in the website, following the above-mentioned approval. This research was performed according to the tenets of the Declaration of Helsinki.

\section{Participants}


Patient data were obtained from the University of Tokyo database. GCD and LCD patients who underwent AS-OCT imaging, in addition to routine examinations, such as slit-lamp microscopy, fundus examinations, and measurements of BCVA, were included. Similarly, patients who had no corneal disease, visual impairment, and other ocular diseases (BCVA of 20/20 or higher), and those were age-matched were included as normal controls. Patients with inaccurate diagnosis, corneal epithelial defects, corneal scarring, and a history of corneal surgery, such as phototherapeutic keratectomy (PTK) and corneal transplantation, which can affect the corneal surface, were excluded.

Two corneal specialists (YA and TM) identified dystrophy phenotypes and subtypes according to the International Classification of Corneal Dystrophies (IC3D) using the clinical information in the medical records. ${ }^{3}$ Then, 4 eyes of 2 patients with GCD type 1 (GCD1), 66 eyes of 35 patients with GCD type 2 (GCD2), 11 eyes of 7 patients with LCD type 1 (LCD1), 11 eyes of 6 patients with LCD type IIIA (LCD3A), and 13 eyes of 8 patients with LCD type IV (LCD4) were included in the final analysis. In total, 32 eyes of 23 controls were also included. Patients with LCD2 were excluded because the condition was different from TGFBI dystrophy. ${ }^{3}$ Patients with other vision-affecting diseases were not included in the analysis of the association between BCVA and Fourier components ( $\mathrm{n}=9$ eyes with GCD [cataract, glaucoma, and diabetic retinopathy] and $n=6$ eyes with LCD [cataract, age-related macular degeneration, and central nervous system disorder]).

\section{Oct Assessment}

All AS-OCT images were obtained using SS-1000 CASIA or SS-2000 CASIA2 (Tomey Corporation, Inc., Aichi, Japan). Corneal shape analysis was performed using two different layered assessments: axial power map of the anterior and posterior surfaces. Values within 3-mm diameter were analyzed. Via a Fourier harmonic analysis, corneal dioptric data were expanded into four components: spherical, regular astigmatism, asymmetry, and higher-order irregularity.

\section{Statistical Analysis}

First, we compared the Fourier components between the phenotypes and control eyes. Second, the association between BCVA and the components was analyzed. Next, similar analyses were performed according to each subtype per phenotype.

TGFBI dystrophy, as is well known, develops bilaterally. ${ }^{1-3}$ Therefore, we used the multivariate linear mixed-effects model in which the random effect was the subjects, with adjusting for age. The model adjusts for the hierarchical structure of the data, modeling in a way in which measurements are grouped within subjects to reduce the possible bias of including both eyes of one patient. ${ }^{24,25}$ This was followed by Dunnett's test for multiple comparisons when comparing the values between each sub-group in the phenotypes and the control. ${ }^{26} \mathrm{~A} p$ value of 0.05 was considered statistically significant. All analyses were performed using R 4.0.2. (R Foundation for Statistical Computing, Vienna, Austria)

\section{Data Availability}

All data generated or analysed during this study are included in this published article.

\section{Declarations}

\section{Author contributions}

T.O. and T.U. contributed for the design of the work. Y.A. and T.O. contributed for drafting the manuscript. Y.A. contributed for the data acquisition. T.O. and R.A. analyzed the data. K.K., T.T., J.Y., S.Y. and T.M. contributed for the supervision of the study. All authors contributed to the final approval of the version published.

\section{Additional Information}

The authors declare no competing interests.

\section{References}


1 Soh, Y. Q. et al. Corneal dystrophies. Nat Rev Dis Primers 6, 46, doi:10.1038/s41572-020-0178-9 (2020).

2 Weiss, J. S. et al. The IC3D classification of the corneal dystrophies. Cornea 27 Suppl 2, S1-83, doi:10.1097/IC0.0b013e31817780fb (2008).

3 Weiss, J. S. et al. IC3D classification of corneal dystrophies-edition 2. Cornea 34, 117-159, doi:10.1097/IC0.0000000000000307 (2015).

4 Klintworth, G. K. Corneal dystrophies. Orphanet J Rare Dis 4, 7, doi:10.1186/1750-1172-4-7 (2009).

5 Sacchetti, M. et al. Pathophysiology of Corneal Dystrophies: From Cellular Genetic Alteration to Clinical Findings. J Cell Physio/ 231, 261-269, doi:10.1002/jcp.25082 (2016).

6 Lakshminarayanan, R. et al. Clinical and genetic aspects of the TGFBI-associated corneal dystrophies. Ocul Surf 12, 234-251, doi:10.1016/j.jtos.2013.12.002 (2014).

7 Han, K. E. et al. Pathogenesis and treatments of TGFBI corneal dystrophies. Prog Retin Eye Res 50, 67-88, doi:10.1016/j.preteyeres.2015.11.002 (2016).

8 Chao-Shern, C. et al. Evaluation of TGFBI corneal dystrophy and molecular diagnostic testing. Eye (Lond) 33, 874-881, doi:10.1038/s41433-019-0346-x (2019).

9 Nielsen, N. S. et al. Biochemical mechanisms of aggregation in TGFBI-linked corneal dystrophies. Prog Retin Eye Res 77, 100843, doi:10.1016/j.preteyeres.2020.100843 (2020).

10 Yagi-Yaguchi, Y. et al. Corneal Higher Order Aberrations in Granular, Lattice and Macular Corneal Dystrophies. PLoS One 11, e0161075, doi:10.1371/journal.pone.0161075 (2016).

11 Yamaguchi, T. et al. A Novel Entity of Corneal Diseases with Irregular Posterior Corneal Surfaces: Concept and Clinical Relevance. Cornea 36 Suppl 1, S53-S59, doi:10.1097/IC0.0000000000001388 (2017).

12 Oshika, T., Okamoto, C., Samejima, T., Tokunaga, T. \& Miyata, K. Contrast sensitivity function and ocular higher-order wavefront aberrations in normal human eyes. Ophthalmology 113, 1807-1812, doi:10.1016/j.ophtha.2006.03.061 (2006).

13 Yamaguchi, T. et al. Feasibility of spherical aberration correction with aspheric intraocular lenses in cataract surgery based on individual pupil diameter. Journal of Cataract \& Refractive Surgery 35, 1725-1733, doi:10.1016/j.jcrs.2009.05.031 (2009).

14 Yamaguchi, T. et al. Effect of spherical aberration on visual function under photopic and mesopic conditions after cataract surgery. Journal of Cataract \& Refractive Surgery 35, 57-63, doi:10.1016/j.jcrs.2008.09.012 (2009).

15 Yamaguchi, T. et al. Comparison of anterior and posterior corneal surface irregularity in Descemet stripping automated endothelial keratoplasty and penetrating keratoplasty. Cornea 29, 1086-1090, doi:10.1097/ICO.0b013e3181d0092c (2010).

16 Yamaguchi, T. et al. The Contribution of the Posterior Surface to the Corneal Aberrations in Eyes after Keratoplasty. Investigative Ophthalmology \& Visual Science 52, 6222-6229, doi:10.1167/iovs.11-7647 (2011).

17 Rudolph, M. et al. Corneal higher-order aberrations after Descemet's membrane endothelial keratoplasty.

18 Tomida, D. et al. Effects of corneal irregular astigmatism on visual acuity after conventional and femtosecond laser-assisted Descemet's stripping automated endothelial keratoplasty.

19 Oie, Y. et al. Fourier Analysis on Regular and Irregular Astigmatism of Anterior and Posterior Corneal Surfaces in Fuchs Endothelial Corneal Dystrophy. American Journal of Ophthalmology 223, 33-41, doi:10.1016/j.ajo.2020.09.045 (2021).

20 Wertheimer, C. M. et al. Corneal optical density in Fuchs endothelial dystrophy determined by anterior segment optical coherence tomography. Eur J Ophthalmol, 1120672120944796, doi:10.1177/1120672120944796 (2020). 
21 Yang, J. et al. Analysis of TGFBI gene mutations in Chinese patients with corneal dystrophies and review of the literature. Mol Vis 16, 1186-1193 (2010).

22 Mashima, Y. et al. Association of autosomal dominantly inherited corneal dystrophies with BIGH3 gene mutations in Japan. Am J Ophthalmol 130, 516-517, doi:10.1016/s0002-9394(00)00571-7 (2000).

23 Munier, F. L. et al. BIGH3 mutation spectrum in corneal dystrophies. Invest Ophthalmol Vis Sci 43, 949-954 (2002).

24 Baayen, R. H., Davidson, D. J. \& Bates, D. M. Mixed-effects modeling with crossed random effects for subjects and items. Journal of Memory and Language 59, 390-412, doi:https://doi.org/10.1016/j.jml.2007.12.005 (2008).

25 Bates, D., Mächler, M., Bolker, B. \& Walker, S. Fitting Linear Mixed-Effects Models Using Ime4. Journal of Statistical Software; Vol 1, Issue 1 (2015), doi:10.18637/jss.v067.i01 (2015).

26 Dunnett, C. W. A Multiple Comparison Procedure for Comparing Several Treatments with a Control. Journal of the American Statistical Association 50, 1096-1121, doi:10.1080/01621459.1955.10501294 (1955).

27 Klintworth, G. K. The molecular genetics of the corneal dystrophies-current status. Front Biosci 8, d687-713, doi:10.2741/1018 (2003).

28 Moshirfar, M., West, W. \& Ronquillo, Y. in StatPearls （StatPearls Publishing

Copyright @ 2021, StatPearls Publishing LLC., 2021).

29 Hieda, O. et al. Clinical outcomes and time to recurrence of phototherapeutic keratectomy in Japan. Medicine (Baltimore) 98 , e16216, doi:10.1097/md.0000000000016216 (2019).

30 Hayashi, K., Kawahara, S., Manabe, S. \& Hirata, A. Changes in Irregular Corneal Astigmatism With Age in Eyes With and Without Cataract Surgery. Invest Ophthalmol Vis Sci 56, 7988-7998, doi:10.1167/iovs.15-18058 (2015).

31 Hidaka, Y. et al. Changes in corneal aberrations after cataract surgery. Jpn J Ophthalmo/60, 135-141, doi:10.1007/s10384-0160431-7 (2016).

32 Koç, M. et al. Effect of corneal biomechanical properties on surgically-induced astigmatism and higher-order aberrations after cataract surgery. Arq Bras Oftalmol 79, 380-383, doi:10.5935/0004-2749.20160108 (2016).

33 Olsen, T., Dam-Johansen, M., Bek, T. \& Hjortdal, J. O. Corneal versus scleral tunnel incision in cataract surgery: a randomized study. J Cataract Refract Surg 23, 337-341, doi:10.1016/s0886-3350(97)80176-9 (1997).

34 Klijn, S., van der Sommen, C. M., Sicam, V. A. \& Reus, N. J. Value of posterior keratometry in the assessment of surgically induced astigmatic change in cataract surgery. Acta Ophthalmo/ 94, 494-498, doi:10.1111/aos.13003 (2016).

35 Rajappa, S. A. \& Bhatt, H. Minimizing surgically induced astigmatism in non-phaco manual small incision cataract surgery by Ushaped modification of scleral incision. Indian J Ophthalmol 68, 2107-2110, doi:10.4103/ijo.IJO_1696_19 (2020).

\section{Figures}

\section{Figure 1}

Representative example of AS-OCT image and topographic map in one case analyzed in this study.

AS-OCT = anterior segment optical coherence tomography 


\section{Figure 2}

Fourier components for comparison between the phenotypes and controls. * indicates statistically significant difference. $\mathrm{GCD}=$ granular corneal dystrophy, $\mathrm{LCD}=$ lattice corneal dystrophy

\section{Figure 3}

Fourier components for comparison between the subtypes and controls. * indicates statistically significant difference.

GCD1 = granular corneal dystrophy type 1, GCD2 = granular corneal dystrophy type $2, \operatorname{LCD} 1$ = lattice corneal dystrophy type 1, LCD3A $=$ lattice corneal dystrophy type 3A, LCD 4 = lattice corneal dystrophy type 4 